\title{
Uso de resina pré-aquecida como material cimentante em restauração indireta: Uma
}

\section{revisão de literatura}

Use of preheated resin as a cementitious material in indirect restoration: A literature review

Uso de resina precalentada como material cementoso en restauración indirecta: Revisión de la

literatura

Recebido: 19/05/2021 | Revisado: 26/05/2021 | Aceito: 28/05/2021 | Publicado: 12/06/2021

João Pedro Silva Teixeira

ORCID: https://orcid.org/0000-0003-4240-6212

Centro Universitário Católica de Quixadá, Brasil

E-mail: Pedro00tri@ hotmail.com

Samara Kelly da Silva Cavalcante ORCID: https://orcid.org/0000-0002-9799-8959 Centro Universitário Católica de Quixadá, Brasil E-mail: samarakelly0804@hotmail.com

Álvaro Tavares Lins Roncolato ORCID: https://orcid.org/0000-0002-3943-0505 Centro Universitário Católica de Quixadá, Brasil E-mail: alvarotavares132002@ gmail.com

Talita Arrais Daniel Mendes ORCID: https://orcid.org/0000-0003-3519-3618 Universidade Federal do Ceará, Brasil

E-mail: talita_arrais@hotmail.com

Ana Carolina Matias Dinelly Pinto ORCID: https://orcid.org/0000-0002-2411-6708 Fundação Oswaldo Cruz, Brasil

E-mail: caroldinelly@hotmail.com

Raynara de Sousa Brito ORCID: https://orcid.org/0000-0003-3602-4357 Centro Universitário Católica de Quixadá, Brasil E-mail: raynarasousa6229@gmail.com

Natasha Muniz Fontes

ORCID: https://orcid.org/0000-0002-0502-0364 Centro Universitário Católica de Quixadá, Brasil E-mail: natashafontes@hotmail.com

Raul Anderson Domingues Alves da Silva ORCID: https://orcid.org/0000-0002-7625-3595 Centro Universitário Católica de Quixadá, Brasil E-mail: raulalves@unicatolicaquixada.edu.br

Pedro Henrique Chaves Isaias ORCID: https://orcid.org/0000-0003-4399-1302 Centro Universitário Católica de Quixadá, Brasil E-mail: pedroisaias@unicatolicaquixada.edu.br

Érika Matias Pinto Dinelly

ORCID: https://orcid.org/0000-0003-2786-3132 Centro Universitário Católica de Quixadá, Brasil E-mail: erikamatias@unicatolicaquixada.edu.br

\begin{abstract}
Resumo
Devido ao grande apelo estético com o decorrer dos anos associado há técnicas minimamente invasivas, a odontologia contemporânea aposta em materiais com ótimas características estéticas e mecânicas, dessa forma os cirurgiões dentistas buscam apostar no uso do pré-aquecimento das resinas compostas como material cimentante para restaurações indiretas, visto que suas propriedades ópticas e mecânicas propõem uma melhor estética e uma melhor adaptação marginal em comparação aos cimentos resinosos. O objetivo deste trabalho é revisar a literatura a cerca das características ópticas e mecânicas dos diferentes materiais utilizados como agente cimentantes em restaurações indiretas, enfatizando a estabilidade de cor ao longo prazo, adaptação marginal, toxicidade, grau de conversão dos monômeros e praticidade clínica. Para tanto, foi realizada uma busca eletrônica na base de dados Sistema Online de Busca e Análise de Literatura Médica (PubMed), utilizando os seguintes descritores em inglês: thermoactivated resin, temperature e dental veneers, cadastrados no Mesh (Medical Subject Headings) combinado entre si pelo operador
\end{abstract}


boleano "AND". Após a delimitação de 10 anos para a busca, foram encontrados 50 artigos. Destes, foram selecionados 13 estudos, com base na leitura de títulos e resumos. Em suma, as resinas pré-aquecidas apresentam ótimas características mecânicas e ópticas, no entanto cabe ao cirurgião-dentista escolher qual técnica é mais viável em sua pratica clínica.

Palavras-chave: Resina termoativada; Temperatura; Facetas dentárias.

\begin{abstract}
Due to the great aesthetic appeal over the years associated with minimally invasive techniques, contemporary dentistry bets on materials with excellent aesthetic and mechanical characteristics, so dentists seek to bet on the use of pre-heating of composite resins as a cementitious material for restorations indirect, since its optical and mechanical properties propose a better aesthetic and a better marginal adaptation compared to resin cements. The objective of this work is to review the literature about the optical and mechanical characteristics of the different materials used as cementing agents in indirect restorations, emphasizing long-term color stability, marginal adaptation, toxicity, degree of conversion of monomers and clinical practicality. For this purpose, an electronic search was performed in the Online Medical Literature Search and Analysis database (PubMed), using the following descriptors in English: thermoactivated resin, temperature and dental veneers, registered in the Mesh (Medical Subject Headings) combined between itself by the Boolean operator "AND". After delimiting the search for 10 years, 50 articles were found. Of these, 13 studies were selected, based on the reading of titles and abstracts. In short, preheated resins have excellent mechanical and optical characteristics, however it is up to the dentist to choose which technique is most feasible in his clinical practice.
\end{abstract}

Keywords: Thermoactivated resin; Temperature; Dental veneers.

\title{
Resumen
}

Debido al gran atractivo estético a lo largo de los años asociado a las técnicas mínimamente invasivas, la odontología contemporánea apuesta por materiales con excelentes características estéticas y mecánicas, por lo que los odontólogos buscan apostar por el uso del precalentamiento de resinas compuestas como material cementoso para restauraciones indirectas, ya que sus propiedades ópticas y mecánicas proponen una mejor estética y una mejor adaptación marginal frente a los cementos resinosos. El objetivo de este trabajo es revisar la literatura sobre las características ópticas y mecánicas de los diferentes materiales utilizados como cementantes en restauraciones indirectas, enfatizando la estabilidad del color a largo plazo, la adaptación marginal, la toxicidad, el grado de conversión de los monómeros y la practicidad clínica. Para ello, se realizó una búsqueda electrónica en la base de datos de Búsqueda y Análisis de Literatura Médica en Línea (PubMed), utilizando los siguientes descriptores en inglés: thermoactivated resin, temperature y dental veneers, registrados en el Mesh (Medical Subject Headings) combinado entre sí por el operador booleano "AND". Tras delimitar la búsqueda por 10 años, se encontraron 50 artículos. De estos, se seleccionaron 13 estudios, en base a la lectura de títulos y resúmenes. En definitiva, las resinas precalentadas tienen unas excelentes características mecánicas y ópticas, sin embargo le corresponde al odontólogo elegir qué técnica es la más factible en su práctica clínica.

Palabras clave: Resina termoactivada; Temperatura; Carillas dentales.

\section{Introdução}

Nos últimos anos, restaurações cerâmicas têm sido frequentemente utilizadas devido à sua excelente estética, biocompatibilidade e estabilidade em longo prazo. Além disso, os cimentos resinosos usados em cimentação de restaurações de cerâmica proporcionam estética relevante, pouca solubilidade em meio bucal, alta resistência de união e propriedades mecânicas consideráveis. Estes materiais resinosos se apresentam como químico, fotopolimerizável ou dual. Os cimentos resinosos fotopolimerizáveis são geralmente o material de escolha sempre que possível devido à sua estabilidade de cor aprimorada e sua capacidade de permitir que o operador controle o tempo de trabalho (El-Korashy, 2010; Magne et al., 2011).

No entanto, o agente de cimentação pode não polimerizar adequadamente devido à atenuação da potência óptica da luz, causada pela interposição de cerâmica entre a unidade de fotopolimerização e o material resinoso (Rathke et al., 2012; Lührs et al., 2014; Alikhasi et al., 2019). A dificuldade em alcançar uma ativação efetiva através de restaurações indiretas levou a uma grande discussão que gira em torno do uso de agentes de cura fotopolimerizáveis. Como esses materiais são ativados apenas fisicamente, há uma diminuição na eficiência de conversão de monômeros em situações de irradiância 
reduzida atingindo o material resinoso. Fatores como opacidade cerâmica, cor e a espessura podem interferir negativamente na transmissão da luz através de materiais restauradores indiretos (Tauböck et al., 2015; Coelho et al., 2019).

A ativação química que ocorre em cimentos duplos pode compensar a atenuação da passagem da luz através de um material cerâmico, melhorando as taxas de conversão de monômeros e as propriedades do material. No entanto, esses agentes duplos contêm mais amina terciária; quando esses componentes oxidam, ocorre uma alteração significativa da cor do agente de cimentação, resultando em estética comprometida do procedimento restaurador. Assim, o uso de agentes de cura fotopolimerizantes deve ser considerado nos casos em que a estética é fundamental, desde que restrita a procedimentos adesivos de restaurações mais finas, como laminados cerâmicos e facetas (Azer et al., 2011; Yang, Silikas \& Watts, 2019).

Estudos anteriores sugeriram que o manuseio de materiais resinosos a temperaturas elevadas, variando de $50{ }^{\circ} \mathrm{C}$ a 60 ${ }^{\circ} \mathrm{C}$ poderia resultar em melhores taxas de conversão, independentemente das condições de polimerização. Com isso, o préaquecimento de compósitos antes da fotoativação geralmente diminui sua viscosidade, melhorando a adaptação marginal e reduzindo a microinfiltração. Além disso, o aumento da temperatura de polimerização aumenta mobilidade radical e monomérica, resultando em maior conversão, que por sua vez pode promover propriedades físicas e mecânicas aprimoradas de compósitos pré-aquecidos, como maior dureza superficial e maior resistência à flexão e resistência à tração (Calheiros et al., 2014; Lima et al., 2018; Chaharom et al., 2020).

O objetivo deste estudo é revisar a literatura acerca da influência do pré-aquecimento e da espessura da cerâmica no grau de conversão, microdureza, densidade de reticulação, resistência à tração final, sorção de água e solubilidade de diferentes materiais resinosos.

\section{Metodologia}

O presente estudo enquadra-se em uma revisão de literatura de natureza qualitativa e com caráter descritivo (Pereira, Shitsuka, Parreira \& Shitsuka, 2018), no qual foram realizadas buscas bibliográficas na base de dados Sistema Online de Busca e Análise de Literatura Médica (PubMed), utilizando os seguintes descritores em inglês: thermoactivated resin, temperature e dental veneers cadastrados no Mesh (Medical Subject Headings) combinado entre si pelo operador booleano "AND”. Após a delimitação de 10 anos para a busca, foram encontrados 50 artigos. Destes, foram selecionados 13 estudos, com base na leitura de títulos e resumos. Foram incluídos artigos em inglês e disponível na íntegra que relatassem a importância da resina termoativada em restaurações indiretas. Foram excluídos artigos que não abortassem o tema proposto, monografias, além de estudos com metodologias incompletas e resultados inconclusivos (Figura 1). 
Figura 1 - Fluxograma para a busca dos artigos.

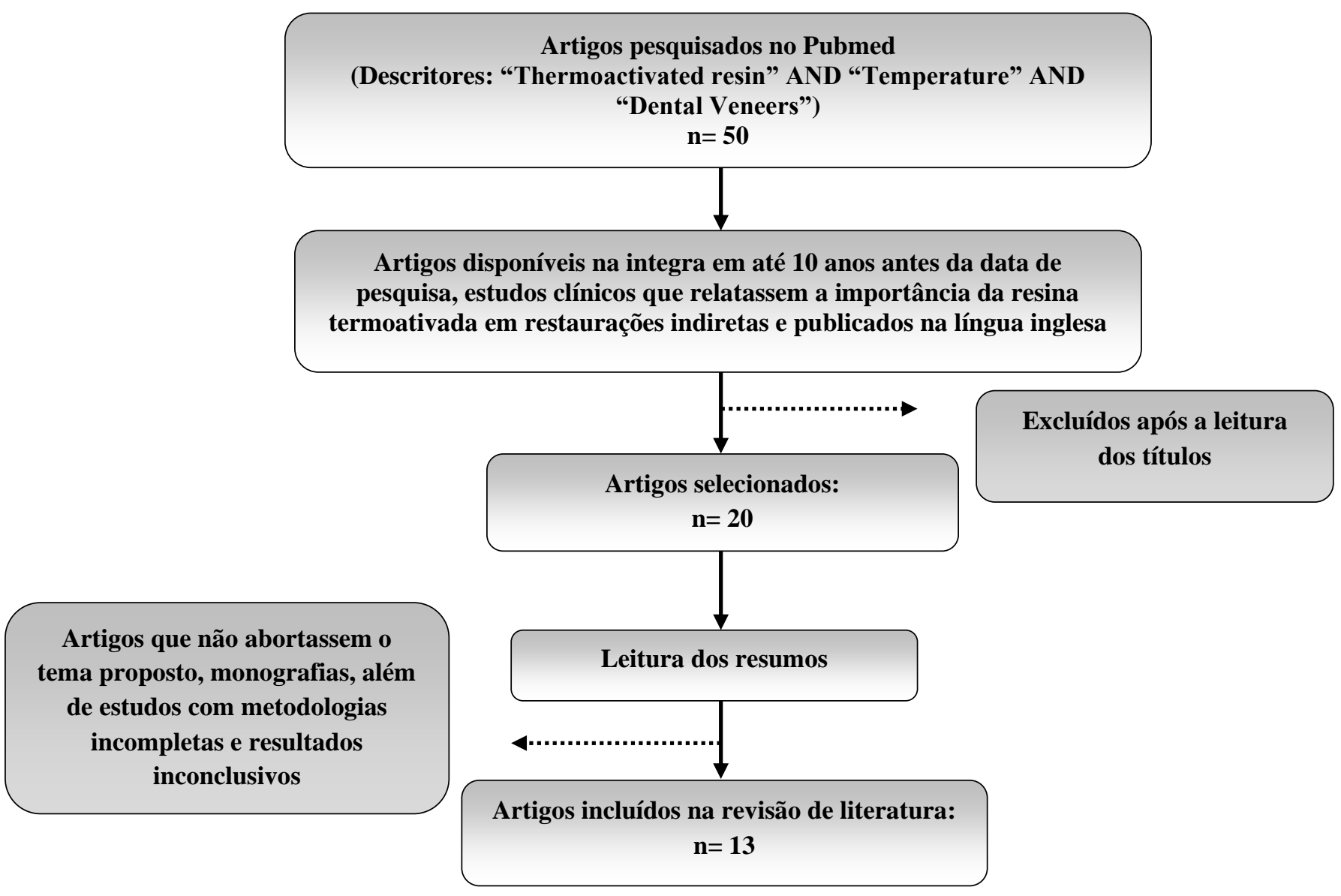

Fonte: Autores (2021).

\section{Resultados}

Após a pesquisa com os descritores thermoactivated resin, temperature e dental veneers na base de dados PubMed, foram encontrados 50 artigos, sendo selecionados 13 estudos. Os resultados encontrados após a leitura crítica dos artigos foram dispostos em uma tabela, com o intuito de facilitar o entendimento (Tabela 1). 
Tabela 1. Estudos que avaliaram a influência do pré-aquecimento e da espessura da cerâmica em diferentes materiais resinosos.

\begin{tabular}{|c|c|c|c|}
\hline Título & Autor/Ano & Metodologia & Resultados \\
\hline $\begin{array}{l}\text { Composite } \\
\text { Pre-Heating: } \\
\text { Effects on } \\
\text { Marginal } \\
\text { Adaptation, } \\
\text { Degree of } \\
\text { Conversion } \\
\text { and } \\
\text { Mechanical } \\
\text { Properties. }\end{array}$ & $\begin{array}{l}\text { Salgado et al., } \\
2010\end{array}$ & $\begin{array}{l}\text { Para a AM, cavidades de classe V ( } 4 \mathrm{~mm} \text { x } 2 \mathrm{~mm} \text { x } 2 \\
\text { mm) foram preparadas em } 40 \text { incisivos bovinos. Foi } \\
\text { aplicado o sistema adesivo Adper Single Bond } 2(3 \mathrm{M} \\
\text { / ESPE). Antes de ser colocado nas cavidades, o } \\
\text { compósito de resina era mantido à temperatura } \\
\text { ambiente }\left(25^{\circ} \mathrm{C} \text { ) ou previamente pré-aquecido a } 68^{\circ}\right. \\
\mathrm{C} \text { no dispositivo Calset. O compósito foi então } \\
\text { polimerizado por } 20 \text { ou } 40 \mathrm{~s} \text { a } 600 \mathrm{~mW} / \mathrm{cm}(12 \text { ou } 24 \\
\mathrm{J} / \mathrm{cm} \text {, respectivamente). A porcentagem de lacunas } \\
\text { foi analisada por microscopia eletrônica de varredura, } \\
\text { após o corte das restaurações e a preparação de } \\
\text { réplicas de resina epóxi. O GC ( } \mathrm{n}=3) \text { foi obtido por } \\
\text { espectroscopia FT-Raman em superfícies compostas } \\
\text { irradiadas e não irradiadas. A RF (n = } 10) \text { foi medido } \\
\text { pelo teste de flexão de três pontos. A KHN (n }=6) \text { foi } \\
\text { medida após } 24 \text { h de armazenamento a seco e } \\
\text { novamente após imersão em solução de etanol a } \\
100 \% \text { por } 24 \text { h, para calcular a densidade de RP. }\end{array}$ & $\begin{array}{l}\text { O compósito pré-aquecido apresentou AM } \\
\text { melhor que os grupos à temperatura } \\
\text { ambiente. Observou-se maior número de } \\
\text { lacunas nos grupos de temperatura ambiente, } \\
\text { independentemente da densidade de energia, } \\
\text { principalmente na parede axial ( } \mathrm{p}<0,05) \text {. O } \\
\text { pré-aquecimento do composto e a densidade } \\
\text { de energia não afetaram as GC, RF e RP (p> } \\
0,05) \text {. }\end{array}$ \\
\hline $\begin{array}{l}\text { Pre-heating of } \\
\text { High- } \\
\text { Viscosity } \\
\text { Bulk-Fill } \\
\text { Resin } \\
\text { Composites: } \\
\text { Effects on } \\
\text { Shrinkage } \\
\text { Force and } \\
\text { Monomer } \\
\text { Conversion }\end{array}$ & $\begin{array}{l}\text { Tauböck et } \\
\text { al., } 2015\end{array}$ & $\begin{array}{l}\text { Foram utilizados quatro materiais compósitos de } \\
\text { enchimento a granel (Tetric EvoCeram Bulk Fill- } \\
\text { TECBF, x-tra fil-XF, QuixFil-QF, SonicFill-SF) e } \\
\text { um composto de resina nano-híbrido convencional } \\
\text { (Tetric EvoCeram-TEC). Os materiais de teste foram } \\
\text { mantidos em temperatura ambiente ou pré-aquecidos } \\
\text { a } 68^{\circ} \mathrm{C} \text { por meio de um dispositivo de aquecimento } \\
\text { comercial, antes de serem fotoativados com uma } \\
\text { unidade de cura por LED por } 20 \mathrm{~s} \text { a } 1170 \mathrm{~mW} / \mathrm{cm} \\
\text { (2). As forças de retração }(\mathrm{n}=5 \text { ) de amostras de } 1,5 \\
\text { mm de espessura foram registradas em tempo real por } \\
15 \text { min dentro de uma câmara com temperatura } \\
\text { controlada a } 25^{\circ} \mathrm{C} \text { (simulando a temperatura intraoral } \\
\text { após a aplicação da barragem de borracha) com um } \\
\text { analisador de estresse personalizado. O grau de } \\
\text { conversão (n }=5 \text { ) foi determinado no fundo de } \\
\text { amostras igualmente grossas (1,5 mm) usando } \\
\text { espectroscopia no infravermelho por transformada de } \\
\text { Fourier. }\end{array}$ & $\begin{array}{l}\text { O pré-aquecimento do composto aumentou } \\
\text { significativamente o grau de conversão do } \\
\text { TECBF, mas não teve efeito na conversão de } \\
\text { monômero dos outros materiais } \\
\text { investigados. Para cada um dos materiais de } \\
\text { teste, o composto pré-aquecido gerou forças } \\
\text { de retração significativamente menores do } \\
\text { que o composto à temperatura ambiente. Nos } \\
\text { dois níveis de temperatura, o TECBF criou as } \\
\text { forças de retração significativamente mais } \\
\text { altas, e o QF causou forças de retração } \\
\text { significativamente mais altas do que o XF e o } \\
\text { TEC. }\end{array}$ \\
\hline $\begin{array}{l}\text { Microshear } \\
\text { Bond Strength } \\
\text { of Preheated } \\
\text { Silorane- and } \\
\text { Methacrylate- } \\
\text { Based } \\
\text { Composite } \\
\text { Resins to } \\
\text { Dentin }\end{array}$ & $\begin{array}{l}\text { Demirbuga et } \\
\text { al., } 2016\end{array}$ & $\begin{array}{l}\text { Os dentes foram divididos aleatoriamente em três } \\
\text { grupos principais: (1) resinas compostas foram } \\
\text { aquecidas até } 68{ }^{\circ} \mathrm{C} ;(2) \text { resfriado a } 4{ }^{\circ} \mathrm{C} \text {; e (3) } \\
\text { controle [temperatura ambiente (TA)]. Cada grupo foi } \\
\text { então subdividido aleatoriamente em quatro } \\
\text { subgrupos de acordo com o sistema adesivo utilizado } \\
\text { [Solobond M (Voco), All Bond SE (Bisco), Clearfil } \\
\text { SE Bond (CSE) (Kuraray), Sistema adesivo de } \\
\text { silorano (SAS) (3M ESPE)]. }\end{array}$ & $\begin{array}{l}\text { O pré-aquecimento de resinas compostas } \\
\text { pode ser uma forma alternativa de aumentar o } \\
\text { MSBS de compósitos em dentina. }\end{array}$ \\
\hline $\begin{array}{l}\text { Effect of Pre- } \\
\text { Heating on } \\
\text { the } \\
\text { Mechanical } \\
\text { Properties of } \\
\text { Silorane- } \\
\text { Based and } \\
\text { Methacrylate- } \\
\text { Based } \\
\text { Composites. }\end{array}$ & $\begin{array}{l}\text { Mohammadi } \\
\text { et al., } 2016\end{array}$ & $\begin{array}{l}\text { Um composto à base de silorano (Silorane) e um } \\
\text { composto à base de metacrilato (Z250) foram pré- } \\
\text { aquecidos a diferentes temperaturas }\left(25,37 \text { e } 68^{\circ} \mathrm{C}\right) \\
\mathrm{e} \text { depois foram testados com os dispositivos } \\
\text { apropriados para cada protocolo de teste. Foram } \\
\text { avaliadas a resistência à flexão, o módulo de } \\
\text { elasticidade e a microdureza de Vickers. ANOVA } \\
\text { bidirecional e post hoc de Tukey foram utilizados } \\
\text { para analisar os dados. }\end{array}$ & $\begin{array}{l}\text { A microdureza e o módulo de elasticidade } \\
\text { aumentaram com o pré-aquecimento, } \\
\text { enquanto os valores de resistência à flexão } \\
\text { não aumentaram significativamente com o } \\
\text { pré-aquecimento. Além disso, o compósito à } \\
\text { base de metacrilato (Z250) apresentou valores } \\
\text { mais altos em comparação ao compósito à } \\
\text { base de silorano (Silorane) em todas as } \\
\text { propriedades testadas. }\end{array}$ \\
\hline
\end{tabular}




\begin{tabular}{|c|c|c|c|}
\hline $\begin{array}{l}\text { Influence of } \\
\text { Ceramic } \\
\text { Laminate } \\
\text { Veneer } \\
\text { Thickness on } \\
\text { Sorption and } \\
\text { Solubility of } \\
\text { Light- Cured } \\
\text { Resin Cement }\end{array}$ & $\begin{array}{l}\text { Mathias et al., } \\
2017 .\end{array}$ & $\begin{array}{l}\text { Vinte e quatro corpos de prova de cimento resinoso } \\
\text { fotopolimerizável ( } 8 \mathrm{~mm} \text { de diâmetro e } 1 \mathrm{~mm} \text { de } \\
\text { espessuraness) foram divididos em quatro grupos de } \\
\text { acordo com a espessura da cerâmica interposta } \\
\text { durante a cura: sem lâmina cerâmicafolheado de nate; } \\
0,7 \mathrm{~mm}, 1,0 \mathrm{~mm} \text { e } 1,3 \mathrm{~mm} \text {. Todas as amostras foram } \\
\text { submetidas ao processo de sorção e solubilidade de } \\
\text { água. oos valores médios de sorção de água e } \\
\text { solubilidade foram comparados pelo teste One-way } \\
\text { ANOVA / Tukey ( } \alpha=0,05) \text {. Pearson'scorrelação foi } \\
\text { realizada para identificar correlações entre sorção de } \\
\text { água e solubilidade, bem como entre } \\
\text { cerâmicaespessura e sorção e solubilidade de água. }\end{array}$ & $\begin{array}{l}\text { Diferenças significativas foram encontradas } \\
\text { nos valores de sorção }(\mathrm{P}<0,05) \text { e solubilidade } \\
(\mathrm{P}<0,01) \text { para ocondições testadas. A sorção } \\
\text { de água e a solubilidade no cimento } \\
\text { fotopolimerizável sob cerâmica mais espessa } \\
\text { foram estatisticamentemaior do que o grupo } \\
\text { de controle. Além disso, houve uma } \\
\text { correlação positiva entre a espessura do } \\
\text { folheado e a absorção de água.ção e } \\
\text { solubilidade do cimento resinoso } \\
\text { fotopolimerizável. }\end{array}$ \\
\hline $\begin{array}{l}\text { The_effect_of } \\
\text { repeated_pre } \\
\text { Heating_of_di } \\
\text { methacrylate }\end{array}$ & $\begin{array}{l}\text { Oskoee et al., } \\
2017 .\end{array}$ & $\begin{array}{l}\text { Neste estudo in vitro, cavidades padrão de Classe V } \\
\text { foram preparadas nas superfícies vestibulares de } 48 \\
\text { incisivos bovinos. Paraprocedimento restaurador, as } \\
\text { amostras foram divididas aleatoriamente em } 2 \text { grupos } \\
\text { com base no tipo de resina composta (grupo 1: di- } \\
\text { compósito de metacrilato [Filtek Z250]; grupo } 2 \text { : } \\
\text { composto de silorano [Filtek P90]) e cada grupo foi } \\
\text { dividido aleatoriamente em } 2 \text { subgrupos com base na } \\
\text { temperatura do composto (A: temperatura ambiente; } \\
\text { B: após } 40 \text { ciclos de pré-aquecimento até } 55^{\circ} \mathrm{C} \text { ). } \\
\text { Marginalas lacunas foram medidas usando um } \\
\text { estereomicroscópio a } 40 \times \text { e analisadas com ANOVA } \\
\text { de duas vias. Comparação inter e intragrupofilhos } \\
\text { foram analisados com testes post-hoc de Tukey. O } \\
\text { nível de significância foi definido em P }<0,05\end{array}$ & $\begin{array}{l}\text { Os gaps máximos e mínimos foram } \\
\text { detectados nos grupos 1-A e 2-B, } \\
\text { respectivamente. Os efeitos do compostotipo } \\
\text { de resina, pré-aquecimento e efeito interativo } \\
\text { dessas variáveis na formação de gap foram } \\
\text { significativos }(\mathrm{P}<0,001) \text {. Post-hoc Tukeyos } \\
\text { testes mostraram maior gap em dimetacrilato } \\
\text { em comparação com resinas compostas de } \\
\text { silorano ( } \mathrm{P}<0,001) \text {. Em cada grupo, valores } \\
\text { de lacunaforam maiores em resinas } \\
\text { compostas em temperatura ambiente em } \\
\text { comparação com resinas compostas após } 40 \\
\text { ciclos de pré-aquecimento }(\mathrm{P}<0,001) \text {. }\end{array}$ \\
\hline $\begin{array}{l}\text { In Vitro } \\
\text { Biocompatibil } \\
\text { ity } \\
\text { of Preheated } \\
\text { Giomer and } \\
\text { Microfilled- } \\
\text { Hybrid } \\
\text { Composite. }\end{array}$ & $\begin{array}{l}\text { Knežević et } \\
\text { al., } 2018\end{array}$ & $\begin{array}{l}\text { Os materiais compósitos (híbrido Gradia Direct } \\
\text { Posterior e Beautifil II) foram aquecidos em uma } \\
\text { unidade de aquecimento Calset a três temperaturas } \\
\text { diferentes (T1: } 37^{\circ} \mathrm{C}, \mathrm{T} 2: 54^{\circ} \mathrm{C}, \mathrm{T} 3: 68^{\circ} \mathrm{C} \text { ). Uma } \\
\text { pequena quantidade de material compósito aquecido } \\
\text { foi colocada em um molde redondo (diâmetro } 6 \mathrm{~mm} \text {; } \\
0,65 \mathrm{~mm} \text { de espessura), coberto com uma folha } \\
\text { Mylar, prensado e polimerizado com a unidade LED } \\
\text { Bluephase. Um grupo de amostras foi polimerizado } \\
\text { diretamente, e o outro grupo através de polímero } \\
\text { reforçado com cerâmica de CAD / CAM (CRP) de } 2 \\
\text { mm de espessura e sobreposição de cerâmica de } \\
\text { dissilicato de lítio CAD / CAM (LDC) por } 20 \text { e } 40 \\
\text { segundos. As amostras polimerizadas foram } \\
\text { colocadas imediatamente após a cura em uma cultura } \\
\text { de células de linfócitos. A viabilidade dos linfócitos } \\
\text { do sangue periférico foi avaliada utilizando uma } \\
\text { técnica de exclusão de corantes por coloração } \\
\text { simultânea com brometo de etídio e laranja de } \\
\text { acridina. }\end{array}$ & $\begin{array}{l}\text { No caso de polimerização de } 20 \text { segundos, o } \\
\text { maior número de polimerização de células } \\
\text { viáveis foi registrado quando os materiais } \\
\text { foram aquecidos a } 37^{\circ} \mathrm{C}(\mathrm{T} 1) \text {, enquanto no } \\
\text { caso de polimerização de } 40 \text { segundos, o } \\
\text { maior número de células viáveis foi } \\
\text { registrado quando os materiais foram } \\
\text { aquecidos a } 54 \circ \mathrm{C} \text { (T2). As amostras } \\
\text { polimerizadas através de sobreposições CAD } \\
\text { / CAM apresentaram menor citotoxicidade do } \\
\text { que as amostras polimerizadas diretamente. }\end{array}$ \\
\hline $\begin{array}{l}\text { Influence of } \\
\text { Pre-Heating } \\
\text { and Ceramic } \\
\text { Thickness on } \\
\text { Physical } \\
\text { Properties of } \\
\text { Luting Agents. }\end{array}$ & $\begin{array}{l}\text { Lima et al., } \\
2018\end{array}$ & $\begin{array}{l}\text { Os materiais RelyX Arc, RelyX Ultimate, RelyX } \\
\text { Veneer e Filtek Z350 Flow foram manipulados em } \\
\text { diferentes temperaturas }\left(23^{\circ} \mathrm{C} \text { ou } 54^{\circ} \mathrm{C}\right) \text {, inseridos } \\
\text { na matriz e fotoativados por discos de cerâmica }(0,75 \\
\text { mm ou } 1,5 \mathrm{~mm}) \text {. Os seguintes testes foram realizados } \\
(\mathrm{n}=8) \text { : grau de conversão, dureza Knoop, densidade } \\
\text { de reticulação, absorção de água, solubilidade e } \\
\text { resistência à tração final. }\end{array}$ & $\begin{array}{l}\text { Quanto à espessura da cerâmica, a cerâmica } \\
\text { mais fina resultou em maiores valores de } \\
\text { dureza Knoop }(\mathrm{p}=0,027) \text {. A temperatura } \\
\text { mais baixa }(23 \circ \mathrm{C}) \text { resultou em maiores } \\
\text { valores de solubilidade }(\mathrm{p}=0,0257) \text { e sorção } \\
\text { de água }(\mathrm{p}=0,0229) \text {. Também houve } \\
\text { diferença estatística entre os materiais: o } \\
\text { RelyX Arc apresentou um maior grau de } \\
\text { conversão e resistência à tração final, seguido } \\
\text { pelo RelyX Veneer, RelyX Ultimate e Filtek } \\
\text { Z350 Flow. Para os testes de dureza Knoop e } \\
\text { densidade de reticulação, o RelyX Ultimate } \\
\text { mostrou os valores mais altos, seguidos pelo } \\
\text { RelyX Arc, RelyX Veneer e Filtek Z350 } \\
\text { Flow. Para sorção e solubilidade em água, o } \\
\text { RelyX Veneer apresentou os valores mais } \\
\text { altos, seguidos pelo RelyX Arc, RelyX }\end{array}$ \\
\hline
\end{tabular}




\begin{tabular}{|c|c|c|c|}
\hline & & & Ultimate e Filtek Z350 Flow. \\
\hline $\begin{array}{l}\text { Response of } \\
\text { Composite } \\
\text { Resins to } \\
\text { Preheating } \\
\text { and the } \\
\text { Resulting } \\
\text { Strengthening } \\
\text { of Luted } \\
\text { Feldspar } \\
\text { Ceramic. }\end{array}$ & $\begin{array}{l}\text { Coelho et al., } \\
2019\end{array}$ & $\begin{array}{l}\text { O módulo de elasticidade, a razão de Poisson e o grau } \\
\text { de conversão do CC foram medidos para três resinas } \\
\text { compostas restauradoras (micro-híbrido Z100; micro- } \\
\text { híbrido imperatriz; nano-híbrido Impress Direct; } \\
\text { omega-supranano estelita) e um cimento resinoso } \\
\text { fotoativado (RelyX Veneer). A viscosidade foi } \\
\text { medida durante uma curva de aquecimento- } \\
\text { resfriamento }\left(25^{\circ} \mathrm{C}-69^{\circ} \mathrm{C}-25^{\circ} \mathrm{C}\right) \text { e também usando } \\
\text { análises isotérmicas a } 25{ }^{\circ} \mathrm{C} \text { e } 699^{\circ} \mathrm{C} \text {. Discos } \\
\text { cerâmicos de feldspato simulando facetas foram } \\
\text { colados com os materiais de cimentação. A força } \\
\text { flexural biaxial, a força característica e o módulo de } \\
\text { Weibull foram calculados nas posições axiais }(\mathrm{z}=0 \text { e } \\
\mathrm{z}=-\mathrm{t} \text { 2) das bicamadas. A espessura do filme foi } \\
\text { medida e a morfologia nas interfaces ligadas foi } \\
\text { observada. }\end{array}$ & $\begin{array}{l}\text { Observou-se uma diminuição gradual da } \\
\text { viscosidade à medida que a temperatura do } \\
\text { reômetro aumentou gradualmente. As } \\
\text { diferenças de viscosidade entre as resinas } \\
\text { compostas foram grandes no início da análise, } \\
\text { mas menores a } 69^{\circ} \text { C. A } 25 \circ \mathrm{C} \text {, os } \\
\text { compósitos eram até } 38 \text { vezes mais viscosos } \\
\text { que o cimento resinoso; a } 69^{\circ} \mathrm{C} \text { a diferença } \\
\text { foi } 5 \text { vezes. A conversão de CC foi } \\
\text { semelhante entre todos os agentes à base de } \\
\text { resina. O cimento resinoso apresentou menor } \\
\text { espessura de filme que os compósitos. Todos } \\
\text { os agentes à base de resina foram capazes de } \\
\text { se infiltrar nos poros da cerâmica na interface } \\
\text { e fortalecer a cerâmica. No entanto, a } \\
\text { magnitude do efeito fortalecedor foi maior } \\
\text { para as resinas compostas pré-aquecidas, } \\
\text { particularmente em z }=-t \text {. } 2 \text {. }\end{array}$ \\
\hline $\begin{array}{l}\text { Influence of } \\
\text { Pre-Heating } \\
\text { Regular Resin } \\
\text { Composites } \\
\text { and Flowable } \\
\text { Composites } \\
\text { on Luting } \\
\text { Ceramic } \\
\text { Veneers With } \\
\text { Different } \\
\text { Thicknesses. }\end{array}$ & $\begin{array}{l}\text { Tomaselli et } \\
\text { al., } 2019\end{array}$ & $\begin{array}{l}\text { Dois experimentos compostos foram preparados (Bis- } \\
\text { GMA / UDMA / BisEMA / TEGDMA), com } \\
\text { diferentes cargas de carga ( } 65 \% \text { ou } 50 \% \text { em peso), } \\
\text { simulando um composto convencional e um fluido. O } \\
\text { fluido (F) foi usado à temperatura ambiente e } \\
\text { convencional, à temperatura ambiente }(\mathrm{C}) \text { ou pré- } \\
\text { aquecido (CPH). Foram preparados discos de } \\
\text { cerâmica com diferentes espessuras }(0,4 \mathrm{~mm}, 0,8 \mathrm{~mm} \text {, } \\
1,5 \mathrm{~mm}) \text {. A espessura da película foi avaliada de } \\
\text { acordo com a ISO } 4049(\mathrm{n}=10) \text {. A resistência da } \\
\text { união ao microcisalhamento }(\mathrm{n}=10) \text { foi avaliada em } \\
\text { esmalte utilizando amostras fotopolimerizadas } \\
\text { usando a cerâmica. O grau de conversão foi avaliado } \\
\text { usando a espectroscopia Raman. A alteração de cor } \\
\text { das restaurações cerâmicas (n }=10) \text { foi avaliada por } \\
\text { espectrofotometria. Os resultados foram submetidos à } \\
\text { ANOVA 2- fatores e ao testepost hoc de Tukey }(\mathrm{a}= \\
5 \%) \text {. Para espessura de filme foi usada um fator } 1 \\
\text { ANOVA }(\mathrm{a}=5 \%) \text {. }\end{array}$ & $\begin{array}{l}\text { O grupo C apresentou maior espessura de } \\
\text { filme; ou CPH produziu uma espessura } \\
\text { semelhante a F. Todos os compostos } \\
\text { apresentaram resistência de união a } \\
\text { microcalhamento semelhante. O grau de } \\
\text { conversão do F foi maior que C e CPH. O } \\
\text { grau de conversão dos compostos } \\
\text { fotoativados em } 0,4 \mathrm{~mm} \text { foi maior que os } \\
\text { compostos fotoativados através de cerâmicas } \\
\text { mais espessas. O grupo C mostra a maior } \\
\text { mudança de cor, enquanto o CPH mostra a } \\
\text { mudança de cor. F. Em conclusão, } \\
\text { compósitos pré-aquecidos, como compósitos } \\
\text { fluídos, parece ser uma alternativa no } \\
\text { potencial para laminados cerâmicos } \\
\text { alimentares. }\end{array}$ \\
\hline $\begin{array}{l}\text { Pre-heating } \\
\text { Effects on } \\
\text { Extrusion } \\
\text { Force, } \\
\text { Stickiness and } \\
\text { Packability of } \\
\text { Resin-Based } \\
\text { Composite. }\end{array}$ & $\begin{array}{l}\text { Yang,Silikas } \\
\text { \& Watts, } 2019\end{array}$ & 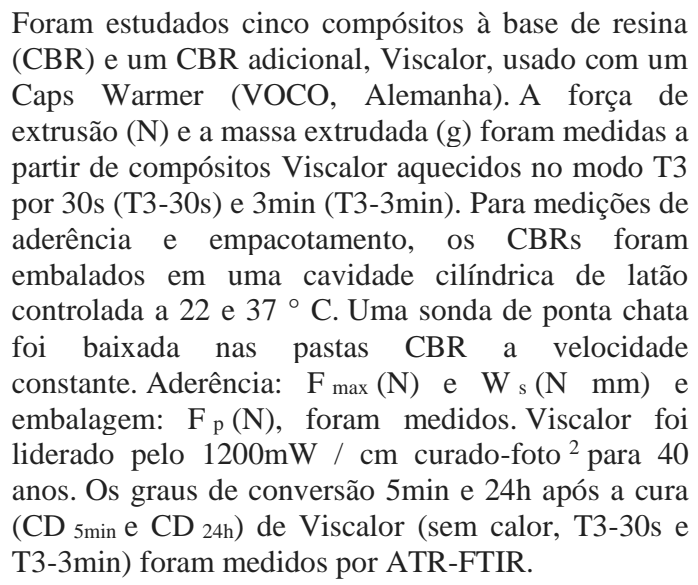 & $\begin{array}{l}\text { A temperatura máxima do Caps Warmer, no } \\
\text { modo T3, atingiu } 68^{\circ} \mathrm{C} \text { em } 20 \text { min. As } \\
\text { temperaturas viscaladas de } 34,5^{\circ} \mathrm{C} \text { e } 60,6^{\circ} \\
\mathrm{C} \text { foram registradas após } 30 \mathrm{~s} \text { e } 3 \mathrm{~min} \text { de pré- } \\
\text { aquecimento, respectivamente. O pré- } \\
\text { aquecimento reduziu significativamente a } \\
\text { força de extrusão e aumentou a massa } \\
\text { extrudada, especialmente após } 3 \\
\text { minutos. GVs variaram em } \mathrm{F}_{\max }, \mathrm{W} \text { s } \\
\mathrm{M}_{\mathrm{p}}(\mathrm{p}<0,05) \text {. A temperatura também afetou } \\
\mathrm{F}_{\max }(\mathrm{p}=0,000), \mathrm{W}_{\mathrm{s}}(\mathrm{p}=0,002) \text { e } \mathrm{F}_{\mathrm{p}}(\mathrm{p}= \\
0,000) \text {. O pré-aquecimento do Viscalor por } \\
30 \mathrm{~s} \text { ou } 3 \text { min não aumentou a DC pós-cura em } \\
5 \text { min ou } 24 \mathrm{~h}, \text { em relação a nenhum pré- } \\
\text { aquecimento }(\mathrm{p}>0,05) \text {. }\end{array}$ \\
\hline $\begin{array}{l}\text { Effect of } \\
\text { Exposure } \\
\text { Time and Pre- } \\
\text { Heating on } \\
\text { the } \\
\text { Conversion } \\
\text { Degree of } \\
\text { Conventional, } \\
\text { Bulk-Fill, } \\
\text { Fiber } \\
\text { Reinforced }\end{array}$ & $\begin{array}{l}\text { Lempel et al., } \\
2019\end{array}$ & $\begin{array}{l}\text { Amostras de dois milímetros de espessura de RBCs } \\
\text { esculpíveis convencionais [FiltekZ250 (FZ)], fluíveis } \\
\text { [Filtek Ultimate Flow (FUF)] e CBRs [Twinky Star } \\
\text { Flow (TS)] modificados por poliácidos e amostras de } \\
\text { quatro milímetros de espessura de RBCs os RBCs de } \\
\text { enchimento a granel [Filtek Bulk Fill Flow (FBF), } \\
\text { Surefil SDR e RBCs esculpidos reforçados com fibra } \\
\text { [EverX Posterior (EX)] foram preparados em um } \\
\text { molde de oito milímetros de profundidade. A } \\
\text { temperatura das hemácias foi predefinida para 25, } 35 \\
\text { e 55 }{ }^{\circ} \text { C. As hemácias foram fotopolimerizadas com }\end{array}$ & $\begin{array}{l}\text { As diferenças em porcentagem GC entre a } \\
\text { parte superior / inferior e o tempo de } \\
\text { exposição recomendado / prolongado foram } \\
\text { significativas para os materiais, exceto SDR } \\
(64,5 / 63,0 \% \text { e } 67,4 / 63,0 \%) \text {. O FUF }(69,0 \% \\
\text { e } 53,4 \% \text { e o TS }(64,9 \% \text { e } 60,9 \%) \text { em } 2 \mathrm{~mm} \\
\text { proporcionaram maior } \% \text { de GC na parte } \\
\text { superior e inferior com o tempo de cura } \\
\text { recomendado, em comparação com os outros } \\
\text { materiais, exceto o SDR. O pré-aquecimento } \\
\text { teve efeito negativo sobre as GC na parte }\end{array}$ \\
\hline
\end{tabular}




\begin{tabular}{|c|c|c|c|}
\hline $\begin{array}{l}\text { and Polyacid- } \\
\text { Modified } \\
\text { Resin } \\
\text { Composites. }\end{array}$ & & $\begin{array}{l}\text { o tempo recomendado e com dupla exposição. As GC } \\
\text { nas partes superior e inferior foram medidas com } \\
\text { espectroscopia micro-Raman. }\end{array}$ & $\begin{array}{l}\text { inferior das hemácias fluidas (FUF: } 48,9 \% \text {, } \\
\text { FBF: } 36,7 \% \text {, SDR: } 43 \% \text {, TS: } 54,7 \% \text { ). O pré- } \\
\text { aquecimento a } 55 \text { C C aumentou } \\
\text { significativamente o\% GC em CBR reforçado } \\
\text { com fibra ( } 75,0 \% \text { na parte superior e } 64,7 \% \\
\text { na parte inferior). }\end{array}$ \\
\hline $\begin{array}{l}\text { Effect of } \\
\text { Preheating on } \\
\text { the } \\
\text { Cytotoxicity of } \\
\text { Bulk-Fill } \\
\text { Composite } \\
\text { Resins. }\end{array}$ & $\begin{array}{l}\text { Chaharom } \\
\text { et al., } 2020\end{array}$ & $\begin{array}{l}\text { Foram utilizados três tipos diferentes de resina } \\
\text { composta, incluindo Tetric N-Ceram Bulk-Fil, Xtrafil } \\
\text { e Xtrabase. De cada resina composta, } 10 \text { amostras } \\
\text { cilíndricas }(5 \mathrm{~mm} \text { de diâmetro e } 4 \mathrm{~mm} \text { de altura) } \\
\text { foram preparadas, com cinco amostras pré-aquecidas } \\
\text { a } 68^{\circ} \mathrm{C} \text { e as outras cinco amostras polimerizadas em } \\
\text { temperatura ambiente }\left(25^{\circ} \mathrm{C}\right) \text {. Vinte e quatro horas } \\
\text { após a polimerização, a citotoxicidade foi avaliada } \\
\text { pelo ensaio MTT em fibroblastos humanos. A análise } \\
\text { estatística dos dados foi realizada com ANOVA } \\
\text { bidirecional e Sidak Post-Hoc. }\end{array}$ & $\begin{array}{l}\text { Não houve diferença estatisticamente } \\
\text { significante entre a porcentagem média de } \\
\text { citotoxicidade em termos de pré-aquecimento } \\
(\mathrm{P}>0,05) \text {, mas a citotoxicidade das resinas } \\
\text { compostas estudadas foi significativamente } \\
\text { diferente }(\mathrm{P}<0,001) \text {. A citotoxicidade da } \\
\text { resina composta Tetric N-Ceram Bulk-fil foi } \\
\text { superior à das outras duas resinas compostas. }\end{array}$ \\
\hline
\end{tabular}

Fonte: Autores (2021).

\section{Discussão}

As resinas compostas são amplamente utilizadas como materiais restauradores em odontologia devido à sua estética, características de manuseio e tempo de trabalho controlado. Caso a polimerização de resinas compostas não ocorra de maneira adequada, os monômeros não reagidos que permanecem na estrutura da resina composta podem ser liberados na cavidade oral após degradação mecânica e química durante o serviço clínico. Em decorrência disso, o pré-aquecimento de resinas compostas não curadas é popular entre os dentistas como uma forma de melhorar os recursos de manuseio durante a colocação. Nesse sentido, o pré-aquecimento de resinas compostas antes da ativação da luz reduz sua viscosidade, e por melhor umedecimento das paredes da cavidade, leva a um aumento na adaptação marginal e uma diminuição na microinfiltração. Além disso, o aumento da temperatura de pré-polimerização resultará em melhor convergência, aumentando a mobilidade de monômeros e radicais (Karacolak et al., 2018).

Deb et al. (2011), através de um estudo in vitro, observaram que aumentar a temperatura para $60{ }^{\circ} \mathrm{C}$ antes da polimerização em resinas compostas posteriores convencionais aumenta significativamente o grau de conversão. Além disso, a conversão aprimorada leva a melhores propriedades físicas e mecânicas, como dureza superficial aprimorada, resistência à flexão e resistência à tração. Além disso, os monômeros não reagidos restantes na estrutura da resina composta serão reduzidos com conversão adicional.

Em concordância com esse estudo, Yang, Silikas \& Watts (2019), avaliaram que o uso de resina pré-aquecida conseguiu uma melhor adaptação marginal devido à termoplasticidade das resinas compostas, ocasionando uma menor força de extrusão, uma melhor aderência e nem afeta no grau de conversão dos monômeros. Entretanto, o pré-aquecimento de resinas fluidas como no caso da Bulk-fill flow (Filtek), apresentou uma serie de defeitos principalmente no grau de conversão dos monômeros, porém quando se pré-aqueceu as resinas compostas como a $\mathrm{Z} 250$ (filtek) à $55^{\circ} \mathrm{C}$ houve um aumento significativo na conversão destes monômeros (Lempel et al., 2019).

Além disso, em outro estudo, Coelho et al. (2019) verificaram que o uso de resina pré-aquecida a $69^{\circ} \mathrm{C}$ apresentou-se até cinco vezes mais viscosas quando comparada aos cimentos resinoso, ocasionando uma melhor infiltração nos poros da interface das cerâmica e consequentemente o seu fortalecimento. Dessa forma, foi observado o aumento das propriedades mecânicas como microdureza e módulo de elasticidade dos compósitos a base de siloreno (Silorane), após submeteram as alterações de temperatura, porém a resistência à flexão não obteve aumentos significativos, diferentes dos compósitos a base de metacrilato (Z250), que apresentou valores mais altos em comparação ao compósito à base de silorano, em todas as propriedades testadas (Mohammadi et al., 2016). 
Corroborando com esses achados, Tauböck et al. (2015) avaliaram quatro materiais compósitos bulk-fill quanto ao seu grau de conversão e formação de força de contração, após serem pré-aquecidos a $68^{\circ} \mathrm{C}$ por meio de um dispositivo de aquecimento comercial. Com base nos resultados, inferiram que o pré-aquecimento do compósito aumenta significativamente o grau de conversão e gera forças de contração significativamente mais baixas do que o composto à temperatura ambiente.

Do mesmo modo, Oskoee e colaboradores em 2017 relataram que o pré-aquecimento de compósitos de resina a base de silorane resultou em uma melhor adaptação e selamento marginal. Dessa forma, o pré-aquecimento de resina composta antes da fotopolimerização teve muitas vantagens potenciais, como o aumento o grau de conversão de monômero e aumento da dureza da superfície. Além disso, estudos anteriores mostraram que o procedimento de pré-aquecimento aumentou o fluxo e melhorou a adaptação da resina nas paredes da cavidade e, portanto, microinfiltração potencialmente reduzida (Demirbuga et al., 2016).

Todavia, Salgado et al. (2010), enfatizou que em condições isotérmicas o pré-aquecimento de composto $\left(60 \mathrm{C}^{\circ}\right)$ é capaz de aumentar a conversão de monômero, conforme a mobilidade molecular é aumentada e a frequência de colisão de espécies reativas é aumentada, tendo assim melhores propriedades mecânicas. Porém, em um ambiente clinico (não isotérmico), o pré-aquecimento do composto a $68 \mathrm{C}^{\circ}$ não melhorou o grau de conversão, resistência flexural ou reticulação do polímero isso devido à queda de temperatura que sofre após dispensá-lo com uma seringa, colocá-lo em uma preparação, contorná-lo e, subsequentemente, polimerizar por luz. Estima-se que quando um composto é aquecido até $60 \mathrm{C}^{\circ}$ e retirado do aparelho, a temperatura diminui $50 \%$ após $2 \min$ e $90 \%$ após $5 \mathrm{~min}$, porém obteve uma adaptação marginal aprimorada. A adaptação marginal foi significativamente melhor na parede axial quando o compósito foi pré-aquecido.

Já os cimentos resinosos podem não polimerizar de maneira satisfatória devido ao decréscimo das propriedades ópticas, gerando uma maior opacidade e consequentemente uma menor passagem de luz, acarretando em uma interposição de cerâmica entre o meio de fotopolimerização e o cimento resinoso gerando lacunas na região marginal principalmente (Rathke et al., 2012). Segundo Lima et al. (2018), a espessura de cerâmica de 0,75mm mostrou-se diferença estatisticamente relevantes em relação a microduzera quando comparada a cerâmicas com 1,5mm de espessura, em relação a solubilidade, sorção de água e resistência a tração final não houve diferença significativa quanto as diferentes espessuras.

Em contrapartida, Mathias et al. (2017), relataram haver diferenças significativas nos valores de sorção e solubilidade em diferentes espessuras de laminados cerâmicos. Em laminados com espessura 0,7mm houve menores valores para sorção e solubilidade de água, porem em laminados com espessura de 1,3mm houve uma diminuição da passagem de luz, acarretando em monômeros residuais, gerando maior sorção de agua e solubilidade, que acaba degradando o cimento resinoso, essa degradação ocorre devido a quebra das ligações químicas dos cimentos resinoso ou amolecimento do material em contato com a água.

Semelhantemente, Tomaselli et al. (2019) inferiram que laminados cerâmicos com espessura menor que $1 \mathrm{~mm}$ gera uma maior conversão dos monômeros em comparação a laminados cerâmicos com espessuras mais espessas. Além disso, acrescentaram que a manipulação de resina pré-aquecida em uma temperatura de $68^{\circ} \mathrm{C}$ conseguiu gerar uma menor geração de forças, ocasionando assim uma menor retração do material.

Outro ponto importante é a respeito da citotoxidade ao pré-aquecimento das resinas composta, segundo Chaharom et al. (2020), o pré-aquecimento desses compósitos não teve efeito estatisticamente significativos em relação à citotoxicidade. Assim, a termoplastificação desses compósitos não causa reações adversas, e sua citocompatibilidade dos componentes liberados não foram afetadas. Em contrapartida, Knežević et al. (2018) relataram que a viabilidade celular foi afetada pela temperatura de pré-aquecimento. Neste estudo foram utilizados linfócitos para investigar viabilidade celular por contato direto com resina composta amostras, a maior viabilidade celular foi relatada em resinas compostas pré-aquecidas até $54{ }^{\circ} \mathrm{C}$ com 40 segundos do tempo de fotopolimerização e a $37^{\circ} \mathrm{C}$ com 20 segundos de fotopolimerização. 


\section{Considerações Finais}

As resinas pré-aquecidas apresentam ótimas características mecânicas e ópticas, no entanto cabe ao cirurgião-dentista escolher qual técnica é mais viável em sua prática clínica, visto que não são todos os compósitos que podem oferecer tais propriedades. Além disso, sua praticidade de manuseio, quando comparado aos cimentos resinosos duais ou fotopolimerizáveis, é menor devido à queda rápida de temperatura após o pré-aquecimento, acarretando em um menor tempo de trabalho.

Desse modo, necessita-se de mais estudos para elucidar a aplicabilidade clínica de resinas pré-aquecidas como material cimentante em restaurações indiretas, uma vez que a dificuldade no controle da temperatura inviabiliza a realização dessa técnica.

\section{Referências}

Alikhasi, M., Monzavi, A., Ebrahimi, H., Pirmoradian, M., Shamshiri, A., \& Ghazanfari, R. (2019). Debonding Time and Dental Pulp Temperature With the Er, Cr: YSGG Laser for Debonding Feldespathic and Lithium Disilicate Veneers. J Lasers Med Sci, 10(3), $211-214$.

Azer, S. S., Rosenstiel, S. F., Seghi, R. R., \& Johnston, W. M. (2011). Effect of substrate shades on the color of ceramic laminate veneers. J Prosthet Dent, $106(3), 179-83$

Calheiros, F. C., Daronch, M., Rueggeberg, F. A., \& Braga, R. R. (2014). Effect of temperature on composite polymerization stress and degree of conversion. Dent Mater, 30(6), 613-8.

Coelho, N. F., Barbon, F. J., Machado, R. G., Boscato, N., \& Moraes, R. R. (2019). Response of composite resins to preheating and the resulting strengthening of luted feldspar ceramic. Dent Mater, 35(10),1430-1438.

Chaharom, M. E., Bahari, M., Safyari, L., Safarvand, H., Shafaei, H., Navimipour J. E., Oskoee A. P., Ajami, A. A., \& Kahnamouei, A. M. (2020). Effect of preheating on the cytotoxicity of bulk-fill composite resins. J Dent Res Dent Clin Dent Prospects, 14(1), 19-25.

Deb, S., Di Silvio, L., Mackler, H. E., \& Millar, B. J. (2011). Pre-warming of dental composites. Dent Mater, 27(4), 51-9.

Demirbuga, S., Ucar, F. I., Cayabatmaz, M., Zorba, Y. O., Cantekin, K., Topçuoğlu, H. S., \& Kilinc, H. I. (2016). Microshear bond strength of preheated silorane- and methacrylate-based composite resins to dentin. Scanning, 38(1), 63-9.

El-Korashy, D. I. (2010). Post-gel shrinkage strain and degree of conversion of preheated resin composite cured using different regimens. Oper Dent, 35(2), $172-9$.

Karacolak, G., Turkun, L.S., Boyacioglu, H., \& Ferracane, J, L. (2018). Influence of increment thickness on radiant energy and microhardness of bulk-fill resin composites. Dent Mater J, 37(2), 206-213.

Knežević, A., Želježić, D., Kopjar, N., Duarte, S. J., \& Tarle, Z. (2018). In Vitro Biocompatibility of Preheated Giomer and Microfilled-Hybrid Composite. Acta Stomatol Croat, 52(4), 286-297.

Lempel, E., Öri, Z., Szalma, J., Lovász, B.V., Kiss, A., Tóth, Á., \& Kunsági-Máté, S. (2019). Effect of exposure time and pre-heating on the conversion degree of conventional, bulk-fill, fiber reinforced and polyacid-modified resin composites. Dent Mater, 35(2), 217-228.

Lima, M. O., Catelan, A., Marchi, G. M., Lima, D. A., Martins, L. R., \& Aguiar, F. H. (2018). Influence of pre-heating and ceramic thickness on physical properties of luting agents. J Appl Biomater Funct Mater, 16(4), 252-259.

Lührs, A. K., De Munck, J., Geurtsen, W., \& Meerbeek, B. V. (2014). Composite cements benefit from light-curing. Dent Mater, 30(3), $292-301$.

Magne, P., Paranhos, M. P., Burnett, L. H. J., Magne, M., \& Belser, U. C. (2011). Fatigue resistance and failure mode of novel-design anterior single-tooth implant restorations: influence of material selection for type III veneers bonded to zirconia abutments. Clin Oral Implants Res, 22(2), 195-200.

Mathias, C., Vitória, L. A., Gomes, R. S., Cavalcanti, A. N., \& Mathias, P. (2017). Influence of Ceramic Laminate Veneer Thickness on Sorption and Solubility of Light-Cured Resin Ce-ment. Int J Dentistry Oral Sci, 4(2), 422-426.

Mohammadi, N., Jafari-Navimipour, E., Kimyai, S., Ajami, A. A, Bahari, M., Ansarin, M., \& Ansarin, M. (2016). Efeito do pré-aquecimento nas propriedades mecânicas de compósitos à base de silorano e metacrilato. Journal of clinic and experimental dentistry, 8 (4), $373-378$.

Oskoee, P. A., Azar, F. P., \& Navimipour, E. J. (2017). The effect of repeated preheating of dimethacrylate and silorane-based composite resins on marginal gap of class V restorations. Journal of dental research, dental clinics, dental prospects, 11(1), 36-42.

Pereira, A. S., Shitsuka, Do. M., Parreira, F. J., \& Shitsuka, R. (2018). Metodologia da Pesquisa Científica-Licenciatura em Computação. Santa Maria.

Rathke, A., Hokenmaier, G., Muche, R., \& Haller, B. (2012). Effectiveness of the bond established between ceramic inlays and dentin using different luting protocols. Journal of Adhesive Dentistry, 14(2), 147-154. 
Research, Society and Development, v. 10, n. 7, e2810716293, 2021

(CC BY 4.0) | ISSN 2525-3409 | DOI: http://dx.doi.org/10.33448/rsd-v10i7.16293

Fróes-Salgado, N. R., Silva, L. M., Kawano, Y., Francci, C., Reis, A., \& Loguercio, A. D. (2010). Composite pre-heating: effects on marginal adaptation, degree of conversion and mechanical properties. Dental materials, 26(9), 908-914.

Tauböck, T. T., Tarle, Z., Marovic, D., \& Attin, T. (2015). Pre-heating of high-viscosity bulk-fill resin composites: effects on shrinkage force and monomer conversion. Journal of dentistry, 43(11), 1358-1364.

Tomaselli, L. D. O., Oliveira, D. C. R. S. D., Favarão, J., Silva, A. F. D., Pires-de-Souza, F. D. C. P., Geraldeli, S., \& Sinhoreti, M. A. C. (2019). Influence of pre-heating regular resin composites and flowable composites on luting ceramic veneers with different thicknesses. Brazilian dental journal, 30(5), $459-466$.

Yang, J., Silikas, N., \& Watts, D. C. (2019). Pre-heating effects on extrusion force, stickiness and packability of resin-based composite. Dental Materials, 35(11), 1594-1602. 